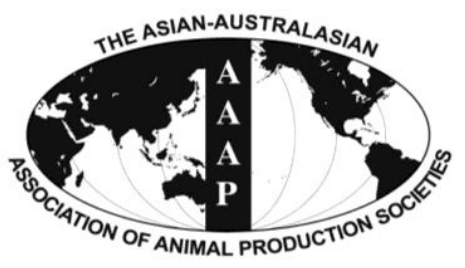

Asian-Aust. J. Anim. Sci.

Vol. 25, No. 6 : 789 - 793

June 2012

www.ajas.info

http://dx.doi.org/10.5713/ajas.2011.11396

\title{
Phosphorylation Status of RNA Polymerase II Carboxyl-terminal Domain in Porcine Oocytes and Early Embryos
}

\author{
Reza K. Oqani, Jin Yu Zhang, Min Gu Lee, Yun Fei Diao and Dong-Il Jin* \\ Department of Animal Science and Biotechnology, Research Center for Transgenic Cloned Pigs, \\ Chungnam National University, Daejeon 305-764, Korea
}

\begin{abstract}
Fertilization of the oocyte commences embryogenesis during which maternally inherited mRNAs are degraded and the embryonic genome is activated. Transcription of embryonic mRNA is initiated by embryonic genome activation (EGA). RNA polymerase II (RNA Pol II) is responsible for the synthesis of mRNAs and most small nuclear RNAs, and consists of 12 subunits, the largest of which characteristically harbors a unique C-terminal domain (CTD). Transcriptional activity of RNA Pol II is highly regulated, in particular, by phosphorylation of serine residues in the CTD. Here, we have shown the presence of RNA Pol II CTD phosphoisoforms in porcine oocytes and preimplantation embryos. The distribution pattern as well as phosphorylation dynamics in germinal vesicles and during embryogenesis differed in developmental stages with these isoforms, indicating a role of RNA Pol II CTD phosphorylation at the serine residue in transcriptional activation during both oocyte growth and embryonic genome activation. We additionally examined the effects of the RNA Pol II inhibitor, $\alpha$-amanitin, on embryo development. Our results show that inhibition of polymerase, even at very early stages and for a short period of time, dramatically impaired blastocyst formation. These findings collectively suggest that the functionality of maternal RNA Pol II, and consequently, expression of early genes regulated by this enzyme are essential for proper embryo development. (Key Words: RNA Polymerase II, Porcine, Oocyte, Embryos, $\alpha$-Amanitin)
\end{abstract}

\section{INTRODUCTION}

During oogenesis, maternal mRNA and proteins are accumulated in oocytes. Embryogenesis commences with fertilization of the oocyte and then some time during embryo development, maternally inherited mRNAs are degraded and the embryonic genome is activated (Schultz, 2002). Following embryonic genome activation (EGA), transcription of embryonic mRNA is initiated. Disruption of these critical events by specific chemicals or environmental factors results in irreversible arrest of embryonic development (Latham, 1999). EGA has been shown to be a species-specific phenomenon. For instance, EGA occurs at the 2-cell stage in mouse (Schultz, 1993) and 4- to 8-cell stages in human, ovine and bovine species (Telford et al., 1990). Previous studies have shown that EGA temporally occurs at the 4-cell stage in porcine embryos (Jarrel et al., 1991). While the molecular mechanism of EGA is not disclosed well, it is expected that a number of genes are

\footnotetext{
* Corresponding Author: Dong-Il Jin. Tel: +82-42-821-5876, Fax: +82-42-822-6712, E-mail: dijin@ @nu.ac.kr Submitted Oct. 31, 2011; Accepted Jan. 18, 2012; Revised Jan. 30, 2012
}

activated and reprogrammed at this stage (Magnani and Cabot, 2009).

RNA polymerase II (RNA Pol II) is responsible for the synthesis of mRNAs and most small nuclear RNAs. RNA Pol II is composed of 12 subunits, of which the largest is characterized by containing a unique $\mathrm{C}$-terminal domain (CTD) that distinguishes the enzyme from other RNA polymerases (Egloff and Murphy, 2008). RNA Pol II functions are highly regulated by modification of the CTD, in particular, phosphorylation of serine residues. Indeed, mRNA synthesis by RNA Pol II requires phosphorylation of CTD at serine 5 (Ser5P) and serine 2 (Ser2P) by the specific kinases, TFIIH and P-TEFb, respectively (Palancade and Bensaude, 2003).

One of the most potent and specific RNA Pol II inhibitors is $\alpha$-amanitin, an antibiotic obtained from the death cap mushroom, Amanita phalloides. This compound affects RNA polymerases in a dose-dependent manner. RNA Pol II is irreversibly inhibited by $10-100 \mu \mathrm{g} / \mathrm{ml}$ of $\alpha$-amanitin. However, at this concentration, RNA Pol I and Pol III are not affected. For inhibition of RNA Pol III, 2- to 4 -fold higher concentrations of $\alpha$-amanitin are required, 
while RNA Pol I is inhibited only in the presence of 1,000fold increased concentration of the drug.

$\alpha$-Amanitin has been extensively used for investigating the sensitivity of living systems to RNA Pol II inhibition. Embryos are reported to be the most sensitive to $\alpha$-amanitin at the time of EGA occurrence. Treatment with this compound inhibits embryonic development beyond the EGA stage (Schultz, 2002; Minami et al., 2007).

The current study was designed to evaluate RNA Pol II expression pattern and function in porcine oocytes and embryos. We further examined the effects of $\alpha$-amanitin treatment just after fertilization on porcine embryo development in vitro.

\section{MATERIALS AND METHODS}

\section{Oocyte collection and in vitro maturation}

Porcine ovaries were collected at a local slaughterhouse. Antral follicles were aspirated manually. Cumulus-oocyte complexes (COCs) were aspirated from 3-6 mm follicles and resuspended in TL-HEPES containing $0.01 \%$ polyvinyl alcohol (PVA). COCs were placed for $22 \mathrm{~h}$ in $500 \mathrm{ml}$ of TCM-199 containing epidermal growth factor, L-cysteine, follicle stimulating hormone (FSH), luteinizing hormone (LH) and follicular fluid at $38.5^{\circ} \mathrm{C}$. Maturation was followed by $22 \mathrm{~h}$ culture in similar conditions without FSH and LH. COCs were denuded by repeated pipetting in TLHEPES containing $0.3 \%$ hyaluronidase in $38.5^{\circ} \mathrm{C}$ and prepared for in vitro fertilization or immunostaining.

In vitro fertilization, embryo culture and drug treatment

After in vitro maturation and denuding, only oocytes with the first polar body extruded were collected. Oocytes were washed 2 times with $\mathrm{mTBM}$ containing $0.1 \%$ BSA as IVF medium and kept in $100 \mu \mathrm{l}$ drops of mTBA/BSA under pre-warmed mineral oil until insemination. Motile sperm from fresh semen were washed two times via centrifugation at 1,200 rpm for $3 \mathrm{~min}$ in $\mathrm{mTBM}$ and incubated for $5 \mathrm{~min}$ at $38.5^{\circ} \mathrm{C}$ for swim-up. Sperm were collected from the surface and added to oocyte-containing IVF droplets. Twenty-five to 30 oocytes were inseminated in each drop. Insemination lasted for $4 \mathrm{~h}$. Oocytes were removed from fertilization drops, washed three times, and cultured in PZM3 medium. $\alpha$-amanitin (A2263) was purchased from Sigma and dissolved in sterile double-distilled water to form $1 \mathrm{mg} / \mathrm{ml}$ stock solution. To determine the effects of $\alpha$-amanitin on pig embryo development, oocytes (during insemination) or embryos (after fertilization) were cultured without or with increasing concentrations of the drug as indicated in the text. The required drug concentrations were prepared from stock solution diluted in mTBM or PZM3. Groups of 25 to 30 embryos were placed in warmed $500 \mu$ of culture medium, covered with mineral oil (Sigma), and cultured under 5\% (v/v) $\mathrm{CO} 2$ at $38.5^{\circ} \mathrm{C}$.

\section{Immunocytochemistry and confocal microscopy}

Oocytes were fixed in PBS-PVA containing 4\% paraformaldehyde and $0.2 \%$ Triton X-100 for $40 \mathrm{~min}$. Fixed oocytes were washed twice in PBS-PVA for $15 \mathrm{~min}$ each and stored overnight in $1 \%$ BSA-supplemented PBS-PVA (BSA-PBS-PVA) at $4^{\circ} \mathrm{C}$. Oocytes were blocked with $10 \%$ goat serum in BSA-PBS-PVA for $45 \mathrm{~min}$, and incubated overnight with primary antibody at $4{ }^{\circ} \mathrm{C}$. The primary antibodies employed included mouse monoclonal antiunphosphorylated RNA Pol II CTD (Clone 8WG16, Upstate) and mouse monoclonal anti-serine 2phosphorylated RNA Pol II CTD (Clone H5, Abcam). After washing three times in BSA-PBS-PVA for $15 \mathrm{~min}$ each, oocytes were incubated for $40 \mathrm{~min}$ at room temperature with conjugated secondary antibodies, FITC-labeled or Texas Red-labeled goat anti-mouse IgG (Santa Cruz Biotechnologies). Next, oocytes were washed three times in BSA-PBS-PVA for $15 \mathrm{~min}$ each, and DNA was stained with propidium iodide ( $400 \mu \mathrm{g} / \mathrm{ml}$; Sigma Chemical Co.) or 4,6diamidino-2-phenylindole (DAPI) (2 $\mu \mathrm{g} / \mathrm{ml}$; Sigma). Following staining, oocytes were washed and mounted on slides with Vectashield mounting medium (Vector Laboratories Inc., Burlingame, CA, USA), and observed under a confocal scanning laser microscope. Images were captured using a Zeiss scanning laser confocal microscope running Zeiss LSM Image Browser software. Serial optical sections (the Z-series) were collected at $1 \mu \mathrm{m}$ intervals, which covered all the nuclear and cytoplasmic regions. The Z-series were stacked, and images depicting staining patterns and intensities of all nuclear and cytoplasmic entities generated. All images in any particular developmental series were acquired using the same laser power output.

\section{RESULTS AND DISCUSSION}

\section{Detection of RNA polymerase II in porcine oocytes}

To determine the presence and localization of RNA Pol II and its phosphoisoforms, we employed immunocytochemistry coupled with confocal microscopy. Using monoclonal antibodies recognizing unphosphorylated RNA Pol II CTD as well as phosphorylated RNA Pol II CTD at serine 2 (Ser2P), we detected both phosphoisoforms in the germinal vesicles of porcine oocytes (Figure 1A). In all cases, unphosphorylated CTD showed an even distribution throughout the nucleoplasm. In all specimens examined, nuclear signals were absent from the nucleolar structure. Phosphorylated CTD was distributed throughout the nucleoplasm, but also formed dot-like structures. 
A

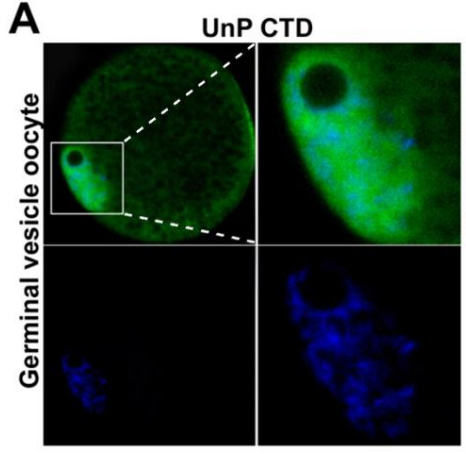

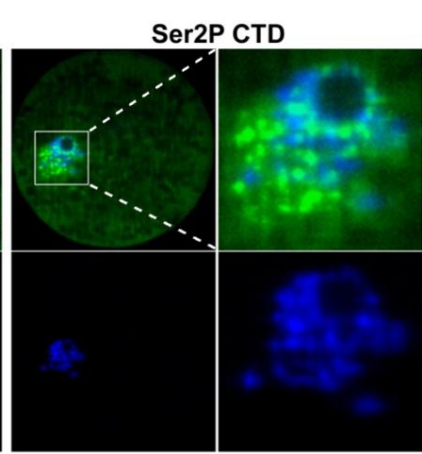

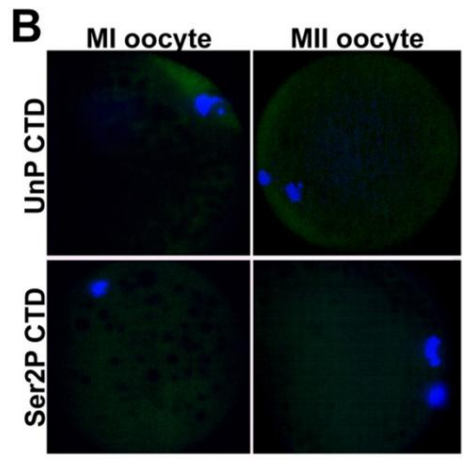

Figure 1. Expression and subcellular localization of RNA Pol II phosphoisoforms in porcine oocytes. (A) The antibody, 8WG16, used in the present study specifically recognized unphosphorylated RNA Pol II CTD. The fluorescence signal is absent in the nucleolar area. DNA is shown in blue and counterstained with DAPI. The H5 antibody specifically recognized Ser2P CTD. This isoform is observed as dot-like structures distributed throughout the germinal vesicle. DNA is shown in blue. Insets show the germinal vesicle areas and enlarged in left. (B) Unphosphorylated CTD in MI and MII pig oocytes. In both MI and MII oocytes, unphosphorylated CTD was not detectable on metaphase chromosomes. Ser2P CTD in MI and MII oocytes. Similar to unphosphorylated CTD, Ser2P was not detectable on MI or MII metaphase chromosomes.

Oocytes were further examined after $22 \mathrm{~h}$ and $44 \mathrm{~h}$ culture in vitro using the same antibodies. In all cases, neither unphosphorylated CTD nor Ser2P CTD was detectable on meiotic MI (22 h) and MII (44 h) chromosomes (Figure 1B). Previous studies have shown that RNA Pol II and other transcription factors are distributed evenly in the nuclei of mammalian cells (Bergman et al., 1995). Moreover, transcriptionally competent cells display dot-like structures in the nucleoplasm. These relatively large, round structures are known as nuclear speckles (Lamond and Spector, 2003). These structures may be reservoirs of active transcription factories and mRNA processing machinery, in particular, pre-mRNA splicing complexes (Spector and Lamond, 2011). CTD phosphorylated at serine 2 is involved in these processes, but not unphosphorylated CTD (Xie et al., 2006). Our experiments confirmed that in pig germinal vesicle oocytes, Ser2P CTD forms speckles, indicating that porcine oocytes are transcriptionally active at this stage. Earlier studies on mitotic cells have also shown that transcription factors are actively disengaged from compacting mitotic chromosomes and involved only after formation of new daughter nuclei (Prasanth et al., 2003). This was verified in our experiments on maturing porcine oocytes. Both unphosphorylated and Ser2P CTD were absent in MI and MII meiotic chromosomes, confirming that oocytes are not transcriptionally active at these stages.

\section{Detection of RNA polymerase II after fertilization}

Next, we examined the presence of RNA Pol II CTD phosphoisoforms in in vitro fertilized porcine embryos. After insemination, zygotes and cleaved embryos were subjected to indirect immunofluorescence staining and confocal microscopy using the same monoclonal antibodies. Unphosphorylated CTD was present at all stages of preimplantation development with equivalent fluorescent signal intensities (Figure 2A). In all the cases examined, signals were limited to nuclear areas and absent in nucleolar structures. The Ser2P CTD isoform was not detectable at the early stages of embryogenesis, but observed shortly after pronuclear formation, and gradually detected after the 4-cell stage (Figure 2B). In all cases, signals were significantly weaker than that of unphosphorylated CTD. Several evidences indicated that unphosphorylated CTD is involved in promoter recognition and pre-initiation complex formation, but not directly correlated with active transcription (Phatnani and Greenleaf, 2006). On the other hand, Ser2P CTD participates in productive transcription and pre-mRNA elongation and splicing. Porcine embryos are transcriptionally inert from germinal vesicle breakdown until EGA at the 4-cell stage (Schoenbeck et al., 1992; Prather et al., 2009). Since activation of transcription requires CTD phosphorylation of RNA Pol II, the observed transcriptionally inactive state in 2- and early 4-cell stage porcine embryos is possibly explained by the absence of CTD kinases and/or mechanism(s) preventing the phosphorylation of CTD. After the 4-cell stage, porcine embryos gradually turn into transcriptionally competent and Ser2P CTD starts to appear in blastomere nuclei, while the fluorescence signal of unphosphorylated CTD remains almost constant.

\section{Effect of $\boldsymbol{\alpha}$-amanitin on embryo development}

To investigate the effect of RNA Pol II inhibition on porcine embryo development in vitro, we performed a series of experiments in which zygotes were exposed to $\alpha$-amanitin at different time-points after fertilization. We examined the formation of blastocysts from fertilized embryos in the presence of increasing drug concentrations 

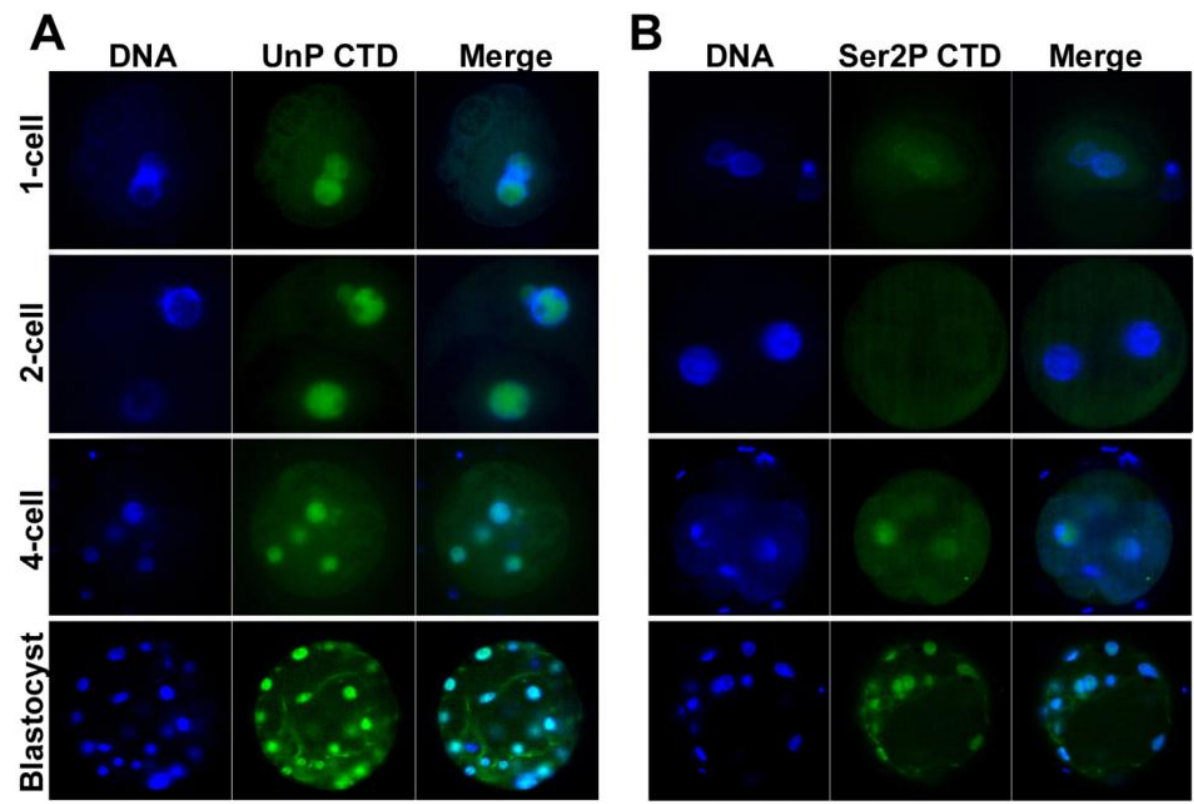

Figure 2. Subcellular localization of RNA Pol II CTD phosphoisoforms during preimplantation development. Immunocytochemical analysis of unphosphorylated CTD (A) and Ser2P CTD (B) in 1-cell, 2-cell, 4-cell and blastocyst embryos. All samples were fixed and stained under similar conditions, and analyzed under the same laser power. DNA was counterstained with DAPI.

at different periods during in vitro culture. Table 1 summarizes the results of these experiments. After insemination, embryos were cultured in absence or presence of $\alpha$-amanitin and monitored for cleavage and blastocyst formation at each day of culture. When porcine embryos were cultured in the presence of $5 \mu \mathrm{g} / \mathrm{ml} \alpha$-amanitin during insemination or for $5 \mathrm{~h}(0$ to $5 \mathrm{~h}$ post-insemination, p.i.) and subsequently cultured in medium devoid of the drug, only

Table 1. Developmental rates of porcine fertilized zygotes in the presence and absence of $\alpha$-amanitin

\begin{tabular}{lcccccl}
\hline $\begin{array}{l}\text { Duration } \\
\text { (h) }\end{array}$ & & \multicolumn{5}{c}{ Concentration $(\mu \mathrm{g} / \mathrm{ml})^{1}$} \\
\cline { 2 - 7 } IVF (4 h) & $\mathrm{T}$ & 3.2 & 0 & 0 & 0 & 0 \\
& $\mathrm{C}$ & 11.5 & 12.0 & 15.4 & 8.9 & 3.3 \\
0 to 5 & $\mathrm{T}$ & 2.1 & 0 & 0 & 0 & 0 \\
& $\mathrm{C}$ & 12.0 & 8.8 & 14.0 & 9.4 & 7.5 \\
5 to 10 & $\mathrm{~T}$ & 0 & 0 & 0 & 0 & 0 \\
& $\mathrm{C}$ & 9.5 & 10.0 & 12.1 & 11.0 & 9.9 \\
24 to 30 & $\mathrm{~T}$ & 0 & 0 & 0 & 0 & 0 \\
& $\mathrm{C}$ & 10.0 & 5.0 & 15.0 & 7.0 & 9.0 \\
48 to 54 & $\mathrm{~T}$ & 2.5 & 0 & 0 & 0 & 0 \\
& $\mathrm{C}$ & 8.2 & 8.8 & 11.3 & 10.2 & 9.9 \\
\hline
\end{tabular}

${ }^{1}$ During insemination and at different time-points after insemination, zygotes were placed in mTBM or PZM3, respectively, containing increasing concentrations of $\alpha$-amanitin, as indicated. In all cases, embryos were washed three times with culture medium to remove all excess drug and transferred to fresh medium without drug. Twenty-five to 30 embryos were treated in each group. The numbers of blastocysts in both control (C) and treated (T) embryos were assessed after 7 days of culture.
$3.2 \%$ or $2.1 \%$ respectively reached the blastocyst stage after 7 days of culture. Under similar conditions, $10.22 \%$ or $10.34 \%$ of fertilized embryos formed blastocysts in absence of $\alpha$-amanitin (control embryos). At increasing drug concentrations $(10,15,20$ and $25 \mu \mathrm{g} / \mathrm{ml})$, no blastocyst formation was observed in treated embryos. Next, we treated embryos with $\alpha$-amanitin at different time-periods after insemination. In one group treated with $5 \mu \mathrm{g} / \mathrm{ml}$ $\alpha$-amanitin during 48 to $54 \mathrm{~h}$ p.i., $2.5 \%$ of fertilized embryos reached the blastocyst stage. In other groups treated during 5 to 10 and 24 to $30 \mathrm{~h}$ p.i. with $5,10,15,20$ and $25 \mu \mathrm{g} / \mathrm{ml}$ of the drug, no blastocyst formation was observed. This finding indicates that even at very early stages of development during which transcription by RNA Pol II is not activated, irreversible blocking of RNA Pol II by $\alpha$-amanitin for a short time $(\sim 5 \mathrm{~h})$ and at low concentrations $(5 \mu \mathrm{g} / \mathrm{ml})$ impairs proper preimplantation development. Previous studies have shown that $\alpha$-amanitin binds to RNA Pol II irreversibly and promotes degradation of the largest subunit (Nguyen et al., 1997). Moreover, RNA Pol II and other transcription factors are maternally contributed in animal embryogenesis (Bellier et al., 1997a; 1997b). Thus, the functionality of maternally provided RNA Pol II, and consequently, expression of genes transcriptionally regulated by this enzyme and inhibited by $\alpha$-amanitin are essential for proper embryo development.

\section{ACKNOWLEDGEMENTS}

This work was supported by a National Research 
Foundation of Korea (NRF) grant funded by the Korean government (MEST) (grant no. 2010-0022263) and the BioGreen 21 Program (grant no. 20070401034031) of the Rural Development Administration, Republic of Korea.

\section{REFERENCES}

Bellier, S., S. Chastant, P. Adenot, M. Vincent, J. P. Renard and O. Bensaude. 1997a. Nuclear translocation and carboxyl-terminal domain phosphorylation of RNA polymerase II delineate the two phases of zygotic gene activation in mammalian embryos. EMBO J. 16:6250-6262.

Bellier, S., M. F. Dubois, E. Nishida, G. Almouzni and O. Bensaude. 1997b. Phosphorylation of the RNA polymerase II largest subunit during Xenopus laevis oocyte maturation. Mol. Cell. Biol. 17:1434-1440.

Bregman, D. B., L. Du, S. van der Zee and S. L. Warren. 1995. Transcription-dependent redistribution of the large subunit of RNA polymerase II to discrete nuclear domains. J. Cell Biol. 129:287-298.

Egloff, S. and S. Murphy. 2008. Cracking the RNA polymerase II CTD code. Trends Genet. 24:280-288.

Jarrell, V. L., B. N. Day and R. S. Prather. 1991. The transition from maternal to zygotic control of development occurs during the 4-cell stage in the domestic pig, Sus scrofa: quantitative and qualitative aspects of protein synthesis. Biol. Reprod. 44:62-68.

Lamond, A. I. and D. L. Spector. 2003. Nuclear speckles: a model for nuclear organelles. Nat. Rev. Mol. Cell Biol. 4:605-612.

Latham, K. E. 1999. Mechanisms and control of embryonic genome activation in mammalian embryos. Int. Rev. Cytol. 193:71-124.

Magnani, L. and R. A. Cabot. 2009. Manipulation of SMARCA2 and SMARCA4 transcript levels in porcine embryos differentially alters development and expression of SMARCA1, SOX2, NANOG, and EIF1. Reproduction 137:23-33.

Minami, N., T. Suzuki and S. Tsukamoto. 2007. Zygotic gene activation and maternal factors in mammals. J. Reprod. Dev. 53:707-715.
Nguyen, V. T., F. Giannoni, M. F. Dubois, S. J. Seo, M. Vigneron, C. Kédinger and O. Bensaude. 1996. In vivo degradation of RNA polymerase II largest subunit triggered by alpha-amanitin. Nucleic Acids Res. 24:2924-2929.

Palancade, B. and O. Bensaude. 2003. Investigating RNA polymerase II carboxyl-terminal domain (CTD) phosphorylation. Eur. J. Biochem. 270:3859-3870.

Phatnani, H. P. and A. L. Greenleaf. 2006. Phosphorylation and functions of the RNA polymerase II CTD. Genes Dev. 20:2922-2936.

Prasanth, K. V., P. A. Sacco-Bubulya, S. G. Prasanth and D. L. Spector. 2003. Sequential entry of components of the gene expression machinery into daughter nuclei. Mol. Biol. Cell 14:1043-1057.

Prather, R. S., J. W. Ross, S. C. Isom and J. A. Green. 2009. Transcriptional, post-transcriptional and epigenetic control of porcine oocyte maturation and embryogenesis. Soc. Reprod. Fertil. 66:165-176.

Schoenbeck, R. A., M. S. Peters, L. F. Rickords, T. T. Stumpf and R. S. Prather. 1992. Characterization of deoxyribonucleic acid synthesis and the transition from maternal to embryonic control in the 4-cell porcine embryo. Biol. Reprod. 47:11181125.

Schultz, R. M. 1993. Regulation of zygotic gene activation in the mouse. Bioessays 15:531-538.

Schultz, R. M. 2002. The molecular foundations of the maternal to zygotic transition in the preimplantation embryo. Hum. Reprod. Update 8:323-331.

Spector, D. L. and A. I. Lamond. 2011. Nuclear speckles. Cold Spring Harb. Perspect. Biol. 1:3.

Telford, N. A., A. J. Watson and G. A. Schultz. 1990. Transition from maternal to embryonic control in early mammalian development: a comparison of several species. Mol. Reprod. Dev. 26:90-100.

Xie, S. Q., S. Martin, P. V. Guillot, D. L. Bentley and A. Pombo. 2006. Splicing speckles are not reservoirs of RNA polymerase II, but contain an inactive form, phosphorylated on serine2 residues of the C-terminal domain. Mol. Biol. Cell 17:17231733. 